\title{
Ending the Global HIV Epidemic Begins at the Individual National Level: An Update from the United States
}

\author{
Robert W. Eisinger ${ }^{1}$; Anthony S. Fauci ${ }^{1, *}$
}

The pathway to achieving an end to the HIV pandemic begins with innovative and sustainable initiatives that optimize for each individual nation the implementation of evidence-based tools in the HIV prevention and treatment toolboxes. This article provides an update on the current global HIV epidemic and describes the current activities associated with the recently announced plan to end the HIV epidemic in the United States.

The latest global statistics on HIV/AIDS showed that in 2019 there were an estimated 38.0 million people living with HIV, 1.7 million new HIV infections, 690,000 deaths from AIDS-related illnesses, and 25.4 million individuals receiving antiretroviral therapy (ART). UNAIDS estimates that in 2019, $81 \%$ of people living with HIV knew their HIV status with $67 \%$ receiving ART, while only $59 \%$ of those individuals receiving ART had undetectable levels of virus (1). In 2014, the UNAIDS issued 3 global targets for achieving HIV treatment scale-up by 2020 that would enable the end of the AIDS epidemic by 2030 . These targets included: $90 \%$ of all people living with HIV infection will know their HIV status; $90 \%$ of all people diagnosed with HIV infection will receive sustained ART; and $90 \%$ of all people receiving ART will have viral suppression (2). However, the 2019 epidemiologic global statistics clearly show that the 9090-90 targets will not be met by the end of 2020. The coronavirus disease 2019 (COVID-19) pandemic further endangers achieving the 90-90-90 targets and may in fact seriously endanger the progress made to date (3). Novel strategies to increase scale up are essential if we are going to successfully achieve the end of HIV/AIDS on a global scale.

Research sponsored and/or conducted by the United States National Institutes of Health $(\mathrm{NIH})$ has and continues to provide much of the essential HIV prevention and treatment tools that need to be implemented to end the HIV pandemic. Landmark prospective clinical studies, including HPTN 052 (4-5), Partners of People on ART-A New Evaluation of the Risks (PARTNER) 1 and 2 (6-7), and
Opposites Attract (8), have demonstrated treatment as prevention (TasP) as a critical tool in the prevention toolbox along with voluntary medical male circumcision, condoms, HIV testing/counseling, screening of the blood supply, and syringe exchange services. A significant tool in preventing HIV infection with ART is pre-exposure prophylaxis (PrEP), with optimal/consistent use of PrEP being about 99\% effective in preventing sexual acquisition of HIV (9). The HIV treatment toolbox includes more than 30 antiretroviral drugs currently approved by the US Food and Drug Administration for treating HIV infection. There are 13 single-drug combinations of 2 or more antiretroviral drugs which can be administered, with limited toxicity, in one-tablet simplified regimens. The consistent use of ART has resulted in near-normal life expectancies for individuals newly diagnosed with HIV (10). While providing ART to everyone living with HIV and providing PrEP to all individuals at-risk of HIV acquisition could theoretically end the epidemic, there is a significant gap that must be bridged in the implementation of the existing HIV prevention and treatment tools (11). There is an underlying need for programs at both a national and global level to optimize the integration of these tools into real-world settings reflecting locally relevant social, cultural, and structural norms.

Transformative programs including the President's Emergency Plan for AIDS Relief (PEPFAR) and the Global Fund to Fight AIDS, Tuberculosis and Malaria continue to maximally implement the existing HIV treatment regimens and prevention strategies throughout the developing world. These efforts are having a significant impact in preventing new HIV infections, providing ART to persons with HIV and achieving viral suppression, and building the health system infrastructure in many countries severely impacted by the HIV pandemic. However, there remains a global HIV treatment gap of roughly 12.4 million persons with HIV who are not receiving ART in 2019 (1), as well as an HIV prevention gap with only an estimated 575,000-580,000 PrEP users 
worldwide-representing a significant underutilization of this effective prevention strategy (12). This latter figure is far below the 2020 UNAIDS target of 3 million PrEP users globally (13). The challenge is to scale-up the uptake of PrEP for at-risk uninfected individuals and provide ART and achieve viral suppression in individuals with HIV. Recent modeling analyses indicate that the COVID-19 pandemic could significantly impact HIV prevention services and disrupt the supply chain of ART resulting in a significant increase in HIV incidence and HIVassociated deaths, especially in high-burden, low- and middle-income nations $(3,14)$. More must be done at the local, regional, and national levels to successfully meet these challenges.

On a national level, the latest statistics from the United States Centers for Disease Control and Prevention (US CDC) indicated that in 2018 there were 1.2 million individuals with HIV, of whom about $14 \%$ were unaware of their HIV infection; 37,968 newly diagnosed HIV infections, with $21 \%$ of these among youths 13-24 years of age and 26\% among adults 25-34 years of age; and the greatest burden of HIV among men who have sex with men (MSM), Blacks/African Americans (15). CDC also reports that in that same year there were 1.2 million individuals in the United States who were at substantial risk for HIV and should be offered PrEP; however, only 219,700 individuals were prescribed PrEP, resulting in a PrEP gap of approximately 900,000 individuals (16).

Facing these daunting statistics, the Trump Administration and key government health officials developed a nationwide initiative entitled, "Ending the HIV Epidemic: A Plan for the United States". This plan was launched in 2019. The goal of this multifaceted "Ending the HIV Epidemic" (EHE) program is to decrease the number of incident HIV infections by $75 \%$ in 5 years and by $90 \%$ by 2030 . The plan is focused on 4 key strategies: 1) diagnose all people with HIV as early as possible; 2) treat people rapidly after diagnosis and effectively reach sustained viral suppression; 3) prevent new HIV transmissions using evidence-based interventions, including PrEP and syringe services programs; and 4) respond quickly to potential outbreaks and emerging clusters of infection (17). The scientific basis for this initiative leverages the significant scientific advances during the past 4 decades that have and continue to provide new and improved HIV diagnostics, therapeutics, and vaccine candidates in the HIV prevention and treatment toolboxes.
The EHE initiative is based on an HIV vulnerability profile including both demographic populations and geographic hotspots. In the United States, demographically $13 \%$ of the population is Blacks/African Americans. CDC reports that $43 \%$ of all new HIV diagnoses are among Blacks/African Americans with $60 \%$ of new HIV infections among MSM in this population and $75 \%$ of new HIV infections in individuals under 35 years of age (15). There also is a geographic concentration as the majority $(>50 \%)$ of new HIV cases reported in 2016 and 2017 were in 48 of the 3007 counties in the United States, Washington D.C., and Puerto Rico, as well as in 7 states with a disproportionate occurrence of HIV in rural areas (17).

Multiple agencies of the United States Department of Health and Human Services (HHS) are cooperating in this initiative including the NIH, US CDC, Health Resources and Services Administration (HRSA), Indian Health Service (IHS), and Substance Abuse and Mental Health Services Administration (SAMHSA). This effort is coordinated by the HHS Office of the Assistant Secretary of Health. Each agency plays a vital role in this multi-HHS agency initiative with CDC intensifying its existing programs in cooperation with local and state health departments for HIV testing, diagnosis, and epidemiologic surveillance and providing rapid linkage to HRSA Ryan White HIV/AIDS Program centers for comprehensive health care. HRSA also accelerates these program centers for HIV treatment and care, as well as providing PrEP for at-risk individuals through its Health Centers Program. The IHS focuses its activities on urban and rural tribal communities. SAMHSA expands use of its Minority AIDS Program and Substance Abuse Prevention and Treatment grants for HIV prevention among individuals with substance abuse or mental illness (17).

The NIH Centers for AIDS Research (CFAR) and AIDS Research Centers (ARCs) serve a critical role in the EHE initiative by informing HHS partners of the best evidence-based state-of-the-art practices resulting from implementation science studies, as well as compiling and disseminating data on the effectiveness of prevention and treatment practices. The 19 CFARs and 7 ARCs are co-located in many of the jurisdictions included in this phase of the initiative (17). These CFAR projects build on close collaborations with local health officials and community groups to design and test implementation strategies reflecting demographic and geographic challenges unique to the local jurisdictions. These studies focus on planning optimal 
delivery of evidence-based HIV prevention interventions, including PrEP for individuals at-risk for HIV, and rapid linkage/relinkage to treatment and health care services for those individuals with HIV (18).

The successful achievement of the EHE initiative in the United States and those in other countries will require optimal implementation of the existing tools in the HIV prevention and treatment toolboxes, as well as the development of new and improved strategies including a safe and effective vaccine and an HIV cure. The ultimate goal of ending the HIV pandemic will require cooperation across the spectrum of the scientific community, industry partners, funders, and civil society at the individual, national, and ultimately, the global levels.

doi: $10.46234 / \mathrm{ccdcw} 2020.163$

\# Corresponding author: Anthony S. Fauci, afauci@niaid.nih.gov.

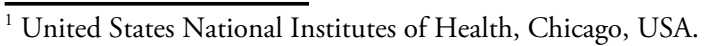

Submitted: July 19, 2020; Accepted: July 29, 2020

\section{REFERENCES}

1. Joint United Nations Programme on HIV/AIDS. Global HIV \& AIDS statistics - 2020 fact sheet. 2020. https://www.unaids.org/en/ resources/fact-sheet. [2020-07-15].

2. Joint United Nations Programme on HIV/AIDS. 90-90-90 An ambitious treatment target to help end the AIDS epidemic. Geneva, Switzerland: Joint United Nations Programme on HIV/AIDS; 2014. https://www.unaids.org/en/resources/documents/2017/90-90-90. [2020-07-15].

3. UNAIDS. Seizing the moment: tackling entrenched inequalities to end epidemics. Geneva, Switzerland; 2020. https://www.philstar.com/ opinion/2020/07/09/2026614/seizing-moment-tackling-entrenchedinequalities-end-epidemics. [2020-07-15].

4. Cohen MS, Chen YQ, McCauley M, Gamble T, Hosseinipour MC, Kumarasamy N, et al. Antiretroviral therapy for the prevention of HIV1 transmission. N Engl J Med 2016;375(9):830 - 9. http://dx.doi.org/ 10.1056/NEJMoa1600693.

5. Cohen MS, Chen YQ, McCauley M, Gamble T, Hosseinipour MC, Kumarasamy N, et al. Prevention of HIV-1 infection with early antiretroviral therapy. N Engl J Med 2011;365(6):493-505. http://dx.doi.org/10.1056/NEJMoa1105243.

6. Rodger AJ, Cambiano V, Bruun T, Vernazza P, Collins S, Degen O, et al. Risk of HIV transmission through condomless sex in serodifferent

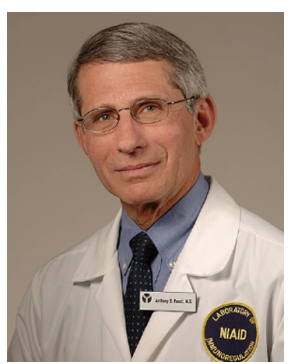

Anthony S. Fauci, M.D.

Director

National Institute of Allergy

and Infectious Diseases

(NIAID)

United States National

Institutes of Health $(\mathrm{NIH})$ gay couples with the HIV-positive partner taking suppressive antiretroviral therapy (PARTNER): final results of a multicentre, prospective, observational study. Lancet 2019;393(10189):2428 - 38 . http://dx.doi.org/10.1016/S0140-6736(19)30418-0.

7. Rodger AJ, Cambiano V, Bruun T, Vernazza P, Collins S, Van Lunzen J, et al. Sexual activity without condoms and risk of HIV transmission in serodifferent couples when the HIV-positive partner is using suppressive antiretroviral therapy. JAMA 2016;316(2):171-81. http://dx.doi.org/10.1001/jama.2016.5148.

8. Bavinton BR, Pinto AN, Phanuphak N, Grinsztejn B, Prestage GP, Zablotska-Manos IB, et al. Viral suppression and HIV transmission in serodiscordant male couples: an international, prospective, observational, cohort study. Lancet HIV 2018;5(8):e438-47. http://dx.doi.org/10.1016/S2352-3018(18)30132-2.

9. US Preventive Services Task Force, Owens DK, Davidson KW, Krist $\mathrm{AH}$, Barry MJ, Cabana $\mathrm{M}$, et al. Preexposure prophylaxis for the prevention of HIV infection: US preventive services task force recommendation statement. JAMA 2019;321(22):2203-13. http://dx.doi.org/10.1001/jama.2019.6390.

10. Marcus JL, Chao CR, Leyden WA, Xu LF, Quesenberry Jr CP, Klein $\mathrm{DB}$, et al. Narrowing the gap in life expectancy between HIV-infected and HIV-uninfected individuals with access to care. J Acquir Immune Defic Syndr 2016;73(1):39 - 46. http://dx.doi.org/10.1097/QAI.0000 000000001014.

11. Eisinger RW, Folkers GK, Fauci AS. Ending the human immunodeficiency virus pandemic: optimizing the prevention and treatment toolkits. Clin Infect Dis 2019;69(12):2212 - 7 . http://dx.doi.org/10.1093/cid/ciz998.

12. PrEP Watch: an AVAC initiative. Global PrEP Tracker 2020. https:// www.prepwatch.org/resource/global-prep-tracker/. [2020-07-15].

13. United Nations General Assembly. Political declaration on HIV and AIDS: on the fast track to accelerating the fight against HIV and to ending the AIDS epidemic by 2030. 2016. https://www.unaids.org/ en/resources/documents/2016/2016-political-declaration-HIV-AIDS. [2020-07-15].

14. Hogan AB, Jewell B, Sherrard-Smith E, Vesga J, Watson OJ, Whittaker C, et al. The potential impact of the COVID-19 epidemic on HIV, TB and malaria in low- and middle-income countries 2020. Imperial College London. http://dx.doi.org/10.25561/78670.

15. Centers for Disease Control and Prevention. HIV Surveillance Report: Diagnoses of HIV infection in the United States and dependent areas, 2018 (Updated). 2020. https:/www.cdc.gov/hiv/library/reports/hivsurveillance/vol-31/index.html. [2020-07-15].

16. Harris NS, Johnson AS, Huang YA, Kern D, Fulton P, Smith DK, et al. Vital signs: status of human immunodeficiency virus testing, viral suppression, and HIV preexposure prophylaxis - United States, 20132018. MMWR Morb Mortal Wkly Rep 2019;68(48):1117-23. http://dx.doi.org/10.15585/mmwr.mm6848e1.

17. Fauci AS, Redfield RR, Sigounas G, Weahkee MD, Giroir BP. Ending the HIV epidemic: a plan for the United States. JAMA 2019; 321(9):844 - 5. http://dx.doi.org/10.1001/jama.2019.1343.

18. NIH bolsters funding for HIV implementation research in high-burden U.S. Areas. https://www.nih.gov/news-events/news-releases/nih-bolstersfunding-hiv-implementation-research-high-burden-us-areas. [2019-09-5].

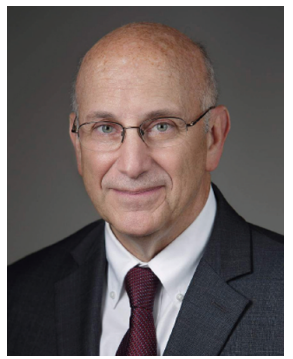

Robert W. Eisinger, Ph.D.

Former Acting Director

Office of AIDS Research

(OAR)

United States National

Institutes of Health (NIH) 